\title{
Families of estimators for ratio and product of study characters using mean and proportion of auxiliary character in presence of non-response
}

\author{
R R Sinha* \\ Department of Mathematics, Dr B R Ambedkar National Institute of Technology, Jalandhar, India \\ *Corresponding author E-mail:raghawraman@gmail.com
}

\begin{abstract}
In this paper, families of estimators for ratio and product of two population means are suggested using proportion and mean of auxiliary character in presence of non-response. The bias and mean square error (MSE) of the proposed families of estimators are obtained up to the first degree of approximation under two different cases. The specified conditions under which the members of proposed families of estimators attain minimum mean square error have been obtained. Theoretical and empirical comparisons based on real data sets are made to show that the suggested families of estimators are more efficient than the relevant estimators such as usual conventional estimator, (Khare \& Sinha 2012 a) estimators and (Sinha 2014) estimators.
\end{abstract}

Keywords: Ratio and Product; Auxiliary Variable; Bias; MSE; Efficiency.

\section{Introduction}

Estimation of ratio and product of two population means is very prevalent in the field of agriculture, socio-economic, medical, forest surveys etc. and fruitfully utilization of auxiliary information at the estimation stage plays an effective role to increase the efficiency of the estimators. Many authors like (Singh 1965, Shah \& Shah 1978, Singh 1982 a, 1982 b, Khare 1987, Srivastativa et al. 1989, Khare 1990, Khare 1991) have considered the problem of estimating ratio and product of two population means using the information of auxiliary character(s) and suggested estimators or classes of estimators.

On the other side, there are many practical situations when auxiliary information is qualitative in nature i.e. auxiliary information is available in the form of attributes and these auxiliary attributes may highly correlated with the study character such as person's height and gender, wool production and breed of sheep, milk production and cow's breed etc. Various authors including (Naik \& Gupta 1996, Jhajj et al. 2006, Singh et al. 2008, Shabbir \& Gupta 2010, Singh \& Solanki 2012, Koyuncu 2012, etc.) have suggested different types of estimators using auxiliary attributes. Further (Khare \& Pandey 2000) and (Khare \& Sinha 2002, 2004, 2007, 2012 a, 2012 b) have proposed some classes of estimators for the ratio and product of two population means using auxiliary character(s) in the presence of non-response. The aim of this paper is to suggest families of estimators for ratio and product of two population means using proportion and mean of auxiliary character in presence of non-response and study their properties.

\section{The proposed estimators}

Let $y_{i},(i=1,2)$ and $x_{i}$ are the main and auxiliary characters under study having non-negative $\mathrm{l}^{\text {th }}$ value $\left(\mathrm{Y}_{\mathrm{il}}, \mathrm{X}_{\mathrm{l}} ; \mathrm{l}=1,2 \ldots \mathrm{N}\right)$ with the population means $\bar{Y}_{i}(i=1,2)$ and $\bar{X}$. Let us consider there is a complete dichotomy on auxiliary character with population proportion $\bar{\emptyset}_{\mathrm{N}}$ in a population with respect to the presence of an attribute $\left(\emptyset_{1}\right.$. Let $\emptyset_{1}$ be the observation of the $\mathrm{l}^{\text {th }}$ unit of attribute $\varnothing$ which takes two values ' 1 ' and ' 0 ' if the $\mathrm{l}^{\text {th }}$ unit possess and does not possesses the attribute respectively. Suppose the whole population is divided into two non overlapping strata of $\mathrm{N}_{1}$ responding and $\mathrm{N}_{2}$ non responding units such that $\mathrm{N}_{1}+\mathrm{N}_{2}=\mathrm{N}$, though they are unknown. Let $\mathrm{n}$ be the size of the sample drawn from the population size $\mathrm{N}$ by using simple random sampling without replacement (SRSWOR) method and it is has been observed that $\mathrm{n}_{1}$ units respond and $\mathrm{n}_{2}$ units do not respond. We have considered here that the responding and non-responding units are same for all the characters and attribute.

Further a subsample of size $r\left(=n_{2} / k, k>1\right)$ from $n_{2}$ non responding units has been drawn by using SRSWOR method after making extra effort. Using the technique of (Hansen \& Hurwitz 1946), the estimators $\bar{y}_{\mathrm{i}}^{*}(\mathrm{i}=1,2), \overline{\mathrm{x}}^{*}$ and $\bar{\emptyset}^{*}$ for the population means $\bar{Y}_{i}(i=1,2), \bar{X}$ and $\bar{\emptyset}$ on the basis of $\left(n_{1}+r\right)$ observations are given by

$$
\begin{aligned}
& \bar{y}_{i}^{*}=\left(\frac{n_{1}}{n}\right) \bar{y}_{i(1)}+\left(\frac{n_{2}}{n}\right) \bar{y}_{i(2)}^{\prime} \\
& \bar{x}^{*}=\left(\frac{n_{1}}{n}\right) \bar{x}_{1}+\left(\frac{n_{2}}{n}\right) \bar{x}_{2}^{\prime}
\end{aligned}
$$

and

$$
\bar{\phi}^{*}=\left(\frac{\mathrm{n}_{1}}{\mathrm{n}}\right) \bar{\phi}_{1}+\left(\frac{\mathrm{n}_{2}}{\mathrm{n}}\right) \bar{\phi}_{2}^{\prime}
$$

where $\left(\overline{\mathrm{y}}_{\mathrm{i}(1)}, \overline{\mathrm{y}}_{\mathrm{i}(2)}^{\prime}\right),\left(\overline{\mathrm{x}}_{1}, \overline{\mathrm{x}}_{2}^{\prime}\right)$ and $\left(\bar{\phi}_{1}, \bar{\phi}_{2}^{\prime}\right)$ are the sample means of the characters $\bar{y}_{i}$, $x$ and $\phi$ based on $n_{1}$ and $r$ units respectively. 
The variances of $\bar{y}_{i}^{*}, \bar{x}^{*}$ and $\bar{\phi}^{*}$ up to the degree of order $\left(n^{-1}\right)$ are given by:

$$
\begin{aligned}
& \mathrm{V}\left(\overline{\mathrm{y}}_{\mathrm{i}}^{*}\right)=\mathrm{V}_{\mathrm{y}_{\mathrm{i}}}^{*} \frac{\mathrm{N}-\mathrm{n}}{\mathrm{Nn}} C_{\mathrm{y}_{\mathrm{i}}}^{2}+\frac{\mathrm{W}_{2}(\mathrm{k}-1)}{\mathrm{n}} C_{\mathrm{y}_{\mathrm{i}}}^{\prime 2}, \mathrm{i}=1,2 \\
& \mathrm{~V}\left(\overline{\mathrm{x}}^{*}\right)=\mathrm{V}_{\mathrm{x}}^{*}=\frac{\mathrm{N}-\mathrm{n}}{\mathrm{Nn}} C_{\mathrm{x}}^{2}+\frac{\mathrm{W}_{2}(\mathrm{k}-1)}{\mathrm{n}} C_{\mathrm{x}}^{\prime^{2}}, \mathrm{i}=1,2
\end{aligned}
$$

and

$$
\mathrm{V}\left(\bar{\emptyset}^{*}\right)=\mathrm{V}_{\varnothing}^{*}=\frac{\mathrm{N}-\mathrm{n}}{\mathrm{Nn}} \mathrm{C}_{\varnothing}^{2}+\frac{\mathrm{W}_{2}(\mathrm{k}-1)}{\mathrm{n}} \mathrm{C}_{\varnothing}^{\prime 2}, \mathrm{i}=1,2
$$

where

$$
\mathrm{C}_{\mathrm{y}_{\mathrm{i}}}^{2}=\frac{\mathrm{S}_{\mathrm{y}_{\mathrm{i}}}^{2}}{\mathrm{Y}_{\mathrm{i}}^{2}}, \mathrm{C}_{\mathrm{x}}^{2}=\frac{\mathrm{S}_{\mathrm{x}}^{2}}{\overline{\mathrm{X}}^{2}}, \mathrm{C}_{\emptyset}^{2}=\frac{\mathrm{S}_{\phi}^{2}}{\bar{\emptyset}^{2}},{\mathrm{C}_{\mathrm{y}_{\mathrm{i}}}^{\prime 2}}^{2} \frac{\mathrm{S}_{\mathrm{y}_{\mathrm{i}}}^{\prime 2}}{\mathrm{Y}_{\mathrm{i}}^{2}}, \mathrm{C}_{\mathrm{x}}^{\prime 2}=\frac{\mathrm{S}_{\mathrm{x}}^{\prime 2}}{\overline{\mathrm{X}}^{2}}, \mathrm{C}_{\emptyset}^{\prime 2}=\frac{\mathrm{S}_{\phi}^{\prime 2}}{\bar{\emptyset}^{2}}
$$

Here $\left(S_{y_{i}}^{2}, S_{x}^{2,}, S_{\varnothing}^{2}\right)$ and $\left(S_{y_{i}}^{\prime 2}, S_{x}^{\prime 2}, S^{\prime 2}{ }_{\varnothing}\right)$ are the mean square of $\left(y_{i}, x, \varnothing\right)$ respectively for the entire and non responding group of population.

Let $\mathrm{u}_{1}\left(=\overline{\mathrm{y}}_{1}^{*} / \overline{\mathrm{y}}_{2}^{*}\right)$ and $\mathrm{u}_{2}\left(=\overline{\mathrm{y}}_{1}^{*} \cdot \overline{\mathrm{y}}_{2}^{*}\right)$ denote the conventional estimators of ratio $\theta_{1}\left(=\overline{\mathrm{Y}}_{1} / \overline{\mathrm{Y}}_{2}\right)$ and product $\theta_{2}\left(=\overline{\mathrm{Y}}_{1}^{*} \cdot \overline{\mathrm{Y}}_{2}^{*}\right)$ of two population means in the presence of non-response.

Now using the auxiliary character with known population mean, (Khare \& Sinha 2012 a) have proposed combined classes of estimators $t_{i j}$ for ratio $\left(\theta_{1}\right)$ and product $\left(\theta_{2}\right)$ of two population means as follows:

$$
t_{i j}=h_{i j}\left(u_{i}, z_{j}\right), i=1,2 ; j=1,2
$$

where $\mathrm{u}_{1}=\left(\overline{\mathrm{y}}_{1}^{*} / \overline{\mathrm{y}}_{2}^{*}\right), \mathrm{u}_{2}=\left(\overline{\mathrm{y}}_{1}^{*} \cdot \overline{\mathrm{y}}_{2}^{*}\right), \mathrm{z}_{1}=\overline{\mathrm{x}}^{*} / \overline{\mathrm{X}}$ and $\mathrm{z}_{2}=\overline{\mathrm{x}} / \overline{\mathrm{X}}$.

Further using the information of auxiliary attribute, (Sinha 2014) suggested classes of estimators for estimating the ratio of the population means $\left(\theta_{1}\right)$ and studied their properties. The classes of estimators proposed by (Sinha 2014) are as follows:

$$
t_{i j}=h_{i j}\left(u_{1}, v_{j}\right), i=3 ; j=1,2
$$

where $\mathrm{v}_{1}=\bar{\emptyset}_{\mathrm{n}}^{*} / \bar{\emptyset}_{\mathrm{N}}$ and $\mathrm{v}_{2}=\bar{\emptyset}_{\mathrm{n}} / \bar{\emptyset}_{\mathrm{N}}$.

Following the lines of (Sinha 2014), we may suggest classes of estimators for estimating the product of the population means $\left(\theta_{2}\right)$ as

$$
t_{i j}=h_{i j}\left(u_{2}, v_{j}\right), i=4 ; j=1,2
$$

and their properties can be studied.

The minimum mean square error of $t_{i j}$ up to the first degree of approximation [i.e. $\mathrm{O}\left(\mathrm{n}^{-1}\right)$ ] under large sample approximation in case of SRSWOR is given by

$$
\begin{aligned}
& \operatorname{MSE}\left(\mathrm{t}_{11}\right)_{\min }=\left(\theta_{1}\right)^{2}\left[\mathrm{M}\left(\mathrm{u}_{1}\right)-\frac{\left[\mathrm{C}_{\mathrm{Rx}}^{*}\right]^{2}}{\mathrm{~V}_{\mathrm{x}}^{*}}\right], \\
& \operatorname{MSE}\left(\mathrm{t}_{12}\right)_{\min }=\left(\theta_{1}\right)^{2}\left[\mathrm{M}\left(\mathrm{u}_{1}\right)-\frac{\left[\mathrm{C}_{\mathrm{Rx}}\right]^{2}}{\mathrm{v}_{\mathrm{x}}}\right], \\
& \operatorname{MSE}\left(\mathrm{t}_{21}\right)_{\min }=\left(\theta_{2}\right)^{2}\left[\mathrm{M}\left(\mathrm{u}_{2}\right)-\frac{\left[\mathrm{C}_{\mathrm{Px}}^{*}\right]^{2}}{\mathrm{~V}_{\mathrm{x}}^{*}}\right], \\
& \operatorname{MSE}\left(\mathrm{t}_{22}\right)_{\min }=\left(\theta_{2}\right)^{2}\left[\mathrm{M}\left(\mathrm{u}_{2}\right)-\frac{\left[\mathrm{C}_{\mathrm{Px}}\right]^{2}}{\mathrm{v}_{\mathrm{x}}}\right], \\
& \operatorname{MSE}\left(\mathrm{t}_{31}\right)_{\min }=\left(\theta_{1}\right)^{2}\left[\mathrm{M}\left(\mathrm{u}_{1}\right)-\frac{\left[\mathrm{C}_{\mathrm{R} \phi}^{*}\right]^{2}}{\mathrm{~V}_{\varnothing}^{*}}\right], \\
& \operatorname{MSE}\left(\mathrm{t}_{32}\right)_{\min }=\left(\theta_{1}\right)^{2}\left[\mathrm{M}\left(\mathrm{u}_{1}\right)-\frac{\left[\mathrm{C}_{\mathrm{R} \emptyset}\right]^{2}}{\mathrm{~V}_{\varnothing}}\right], \\
& \operatorname{MSE}\left(\mathrm{t}_{41}\right)_{\min }=\left(\theta_{2}\right)^{2}\left[\mathrm{M}\left(\mathrm{u}_{2}\right)-\frac{\left[\mathrm{C}_{\mathrm{P} \phi}^{*}\right]^{2}}{\mathrm{~V}_{\varnothing}^{*}}\right]
\end{aligned}
$$

and

$$
\operatorname{MSE}\left(\mathrm{t}_{42}\right)_{\min }=\left(\theta_{2}\right)^{2}\left[\mathrm{M}\left(\mathrm{u}_{2}\right)-\frac{\left[\mathrm{C}_{\mathrm{P} \emptyset}\right]^{2}}{\mathrm{~V}_{\emptyset}}\right] \text {, }
$$

where

$\operatorname{MSE}\left(\mathrm{u}_{1}\right)=\left(\theta_{1}\right)^{2} \mathrm{M}\left(\mathrm{u}_{1}\right)$,

$$
\begin{gathered}
M\left(u_{1}\right)=\left[\frac{N-n}{N n}\left(C_{y_{1}}^{2}+C_{y_{2}}^{2}-2 \rho C_{y_{1}} C_{y_{2}}\right)+\frac{w_{2}(k-1)}{n}\left(C_{y_{1}}^{\prime 2}+C_{y_{2}}^{\prime 2}-\right.\right. \\
\left.\left.2 \rho_{(2)} C_{y_{1}}^{\prime} C_{y_{2}}^{\prime}\right)\right],
\end{gathered}
$$

$\operatorname{MSE}\left(\mathrm{u}_{2}\right)=\left(\theta_{2}\right)^{2} \mathrm{M}\left(\mathrm{u}_{2}\right)$

$$
\mathrm{M}\left(\mathrm{u}_{2}\right)=\left[\frac{\mathrm{N}-\mathrm{n}}{\mathrm{Nn}}\left(\mathrm{C}_{\mathrm{y}_{1}}^{2}+\mathrm{C}_{\mathrm{y}_{2}}^{2}+2 \rho \mathrm{C}_{\mathrm{y}_{1}} \mathrm{C}_{\mathrm{y}_{2}}\right)+\frac{\mathrm{W}_{2}(\mathrm{k}-1)}{\mathrm{n}}\left(\mathrm{C}_{\mathrm{y}_{1}}^{\prime 2}+\mathrm{C}_{\mathrm{y}_{2}}^{\prime 2}+\right.\right.
$$

$$
\begin{aligned}
\mathrm{C}_{\mathrm{R} \emptyset}^{*}=\frac{\mathrm{N}-\mathrm{n}}{\mathrm{Nn}}\left(\rho_{10} \mathrm{C}_{\mathrm{y}_{1}}-\rho_{20} \mathrm{C}_{\mathrm{y}_{2}}\right) \mathrm{C}_{\varnothing} \\
\quad+\frac{\mathrm{W}_{2}(\mathrm{k}-1)}{\mathrm{n}}\left(\rho_{10}^{\prime} \mathrm{C}_{\mathrm{y}_{1}}^{\prime}-\rho_{20}^{\prime} \mathrm{C}_{\mathrm{y}_{2}}^{\prime}\right) \mathrm{C}_{\emptyset}^{\prime},
\end{aligned}
$$

$\mathrm{C}_{\mathrm{Rx}}^{*}=\frac{\mathrm{N}-\mathrm{n}}{\mathrm{Nn}}\left(\rho_{13} \mathrm{C}_{\mathrm{y}_{1}}-\rho_{23} \mathrm{C}_{\mathrm{y}_{2}}\right) \mathrm{C}_{\mathrm{x}}$

$$
+\frac{\mathrm{W}_{2}(\mathrm{k}-1)}{\mathrm{n}}\left(\rho_{13}^{\prime} \mathrm{C}_{\mathrm{y}_{1}}^{\prime}-\rho_{23}^{\prime} \mathrm{C}_{\mathrm{y}_{2}}^{\prime}\right) \mathrm{C}_{\mathrm{x}}^{\prime}
$$

$C_{R \varnothing}=\frac{N-n}{N n}\left(\rho_{10} C_{y_{1}}-\rho_{20} C_{y_{2}}\right) C_{\varnothing}$,

$C_{R x}=\frac{N-n}{N n}\left(\rho_{10} C_{y_{1}}-\rho_{20} C_{y_{2}}\right) C_{\emptyset}$,

$\mathrm{V}(\bar{\varnothing})=\mathrm{V}_{\varnothing}=\frac{\mathrm{N}-\mathrm{n}}{\mathrm{Nn}} \mathrm{C}_{\varnothing}^{2}$

and

$V(\bar{x})=V_{x}=\frac{N-n}{N n} C_{\varnothing}^{2}$

Here $\left(\rho, \rho_{13}, \rho_{23}\right)$ and $\left(\rho_{(2)}, \rho_{13}^{\prime}, \rho_{23}^{\prime}\right)$ are the correlation coefficients between $\left(\mathrm{y}_{1}, \mathrm{y}_{2}\right),\left(\mathrm{y}_{1}, \mathrm{x}\right),\left(\mathrm{y}_{2}, \mathrm{x}\right)$ for the entire and non responding group the population while $\left(\rho_{10}, \rho_{20}, \rho_{30}\right)$ and $\left(\rho_{10}^{\prime}, \rho_{20}^{\prime}, \rho_{30}^{\prime}\right)$ are the biserial correlation coefficients between $\left(\mathrm{y}_{1}, \varnothing\right),\left(\mathrm{y}_{2}, \emptyset\right),(\mathrm{x}, \varnothing)$ for the entire and non responding group of population.

Now following the strategies of (Khare \& Sinha 2012 a, Sinha 2014 and Sinha \& Kumar 2014), we have suggested two wider families of estimators for estimating the ratio and product of two population means using proportion and mean of auxiliary character for two different cases as follows:

Case I - In this case we assumed that there is incomplete information on study characters $\mathrm{y}_{\mathrm{i}}(\mathrm{i}=1,2)$ as well as auxiliary character $\mathrm{x}$ due to non-response, however the population proportion $\left(\bar{\emptyset}_{\mathrm{N}}\right)$ and mean $(\overline{\mathrm{X}})$ are known in advance. We observe that $\mathrm{n}_{1}$ units respond and $n_{2}\left(=n-n_{1}\right)$ units do not respond for $y_{i}, x$ and $\emptyset$ in the sample of size $n$. therefore the proposed families of estimators for estimating ratio and product of two population in presence of non-response is

$$
T_{1 j}=u_{j} f_{1 j}\left(v_{1}, z_{1}\right), j=1,2
$$

such that

$$
\mathrm{f}_{1 \mathrm{j}}(1,1)=1 \text {, }
$$

where

$$
\mathrm{u}_{1}=\overline{\mathrm{y}}_{1}^{*} / \overline{\mathrm{y}}_{2}^{*}, \mathrm{u}_{2}=\overline{\mathrm{y}}_{1}^{*} \cdot \overline{\mathrm{y}}_{2}^{*}, \quad \mathrm{v}_{1}=\bar{\emptyset}_{\mathrm{n}}^{*} / \bar{\emptyset}_{\mathrm{N}}, \quad \text { and } \quad \mathrm{z}_{1}=\overline{\mathrm{x}}^{*} / \overline{\mathrm{X}} \text {. }
$$


Case II - In this case we assumed that there is incomplete information on study characters $y_{i}(i=1,2)$ due to non-response while complete information on auxiliary character $\mathrm{x}$ [see (Rao 1986)], however the population proportion $\left(\bar{\emptyset}_{\mathrm{N}}\right)$ and mean $(\overline{\mathrm{X}})$ are known in advance. We scrutinize that $\mathrm{n}_{1}$ units respond and $\mathrm{n}_{2}\left(=\mathrm{n}-\mathrm{n}_{1}\right)$ units do not respond for $\mathrm{y}_{\mathrm{i}}, \mathrm{x}$ and $\varnothing$ in the sample of size $\mathrm{n}$. therefore the proposed families of estimators for estimating ratio and product of two population means in presence of non-response is

$$
T_{2 j}=u_{j} f_{2 j}\left(v_{2}, z_{2}\right), j=1,2
$$

such that

$$
\mathrm{f}_{2 \mathrm{j}}(1,1)=1 \text {, }
$$

where

$$
\mathrm{u}_{1}=\overline{\mathrm{y}}_{1}^{*} / \overline{\mathrm{y}}_{2}^{*}, \quad \mathrm{u}_{2}=\overline{\mathrm{y}}_{1}^{*} \cdot \overline{\mathrm{y}}_{2}^{*}, \mathrm{v}_{2}=\bar{\emptyset}_{\mathrm{n}} / \bar{\emptyset}_{\mathrm{N}}, \text { and } \mathrm{z}_{2}=\overline{\mathrm{x}} / \overline{\mathrm{X}} .
$$

To study the properties of the proposed families of estimators $\mathrm{T}_{1 \mathrm{j}}$ and $\mathrm{T}_{2 \mathrm{j}}$, we may combine both of them and rewritten as

$$
T_{i j}=u_{j} f_{i j}\left(v_{i}, z_{i}\right), i=1,2 ; j=1,2 .
$$

The function $f_{i j}\left(v_{i}, z_{i}\right)$ is a function of sample values, so for the expansion of $f_{i j}\left(v_{i}, z_{i}\right)$ we assume that it satisfies the following regularity conditions:

i) whatever be the sample chosen, $\left(v_{i}, z_{i}\right)$ assumes values in a bounded, closed convex subset $\mathrm{D}$ of the two dimensional real space containing point $(1,1)$

ii) the function $\mathrm{f}_{\mathrm{ij}}\left(\mathrm{v}_{\mathrm{i}}, \mathrm{z}_{\mathrm{i}}\right)$ is continuous and bounded in D and

iii) the first and second partial derivative of $f_{i j}\left(v_{i}, z_{i}\right)$ exist and are continuous as well as bounded in D.

\section{The bias and mean square error (MSE)}

On account of regularity conditions imposed on $\mathrm{f}_{\mathrm{ij}}\left(\mathrm{v}_{\mathrm{i}}, \mathrm{z}_{\mathrm{i}}\right)$, it may be seen that the bias (B) and the mean square error (MSE) of the estimator $T_{i j}$ will always exist. Expanding the function $f_{i j}\left(v_{i}, z_{i}\right)$ about the point $(1,1)$ using Taylor's series up to the second order partial derivatives we have

$$
\begin{aligned}
& \mathrm{T}_{\mathrm{ij}}=\mathrm{u}_{\mathrm{j}}\left[\mathrm{f}_{\mathrm{ij}}(1,1)+\left(\mathrm{v}_{\mathrm{i}}-1\right) \mathrm{f}_{\mathrm{ij}}^{(1)}+\left(\mathrm{z}_{\mathrm{i}}-1\right) \mathrm{f}_{\mathrm{ij}}^{(2)}\right. \\
&+\frac{1}{2}\left\{\left(\mathrm{v}_{\mathrm{i}}-1\right)^{2} \mathrm{f}_{\mathrm{ij}}^{(11)}\left(\mathrm{v}_{\mathrm{i}}^{\oplus} \mathrm{z}_{\mathrm{i}}^{\oplus}\right)+2\left(\mathrm{v}_{\mathrm{i}}-1\right)\left(\mathrm{z}_{\mathrm{i}}-1\right) \mathrm{f}_{\mathrm{ij}}^{(12)}\left(\mathrm{v}_{\mathrm{i}}^{\oplus} \mathrm{z}_{\mathrm{i}}^{\oplus}\right)\right. \\
&\left.\left.+\left(\mathrm{z}_{\mathrm{i}}-1\right)^{2} \mathrm{f}_{\mathrm{ij}}^{(22)}\left(\mathrm{v}_{\mathrm{i}}^{\oplus} \mathrm{z}_{\mathrm{i}}^{\oplus}\right)\right\}\right]
\end{aligned}
$$

Using the equations given in equations (27) and (29), we have

$$
\begin{aligned}
\mathrm{T}_{\mathrm{ij}}=\mathrm{u}_{\mathrm{j}}\left[1+\left(\mathrm{v}_{\mathrm{i}}-1\right) \mathrm{f}_{\mathrm{ij}}^{(1)}+\left(\mathrm{z}_{\mathrm{i}}-1\right) \mathrm{f}_{\mathrm{ij}}^{(2)}\right. & \\
+\frac{1}{2}\left\{\left(\mathrm{v}_{\mathrm{i}}-1\right)^{2} \mathrm{f}_{\mathrm{ij}}^{(11)}\left(\mathrm{v}_{\mathrm{i}}^{\oplus} \mathrm{z}_{\mathrm{i}}^{\oplus}\right)\right. & +2\left(\mathrm{v}_{\mathrm{i}}-1\right)\left(\mathrm{z}_{\mathrm{i}}-1\right) \mathrm{f}_{\mathrm{ij}}^{(12)}\left(\mathrm{v}_{\mathrm{i}}^{\oplus} \mathrm{z}_{\mathrm{i}}^{\oplus}\right) \\
& \left.\left.+\left(\mathrm{z}_{\mathrm{i}}-1\right)^{2} \mathrm{f}_{\mathrm{ij}}^{(22)}\left(\mathrm{v}_{\mathrm{i}}^{\oplus} \mathrm{z}_{\mathrm{i}}^{\oplus}\right)\right\}\right]
\end{aligned}
$$

where

$$
\begin{aligned}
& \mathrm{f}_{\mathrm{ij}}^{(1)}=\left(\frac{\partial \mathrm{f}_{\mathrm{ij}}}{\partial \mathrm{v}_{\mathrm{i}}}\right)_{(1,1)}, \mathrm{f}_{\mathrm{ij}}^{(2)}=\left(\frac{\partial \mathrm{f}_{\mathrm{ij}}}{\partial \mathrm{z}_{\mathrm{i}}}\right)_{(1,1)}, \mathrm{f}_{\mathrm{ij}}^{(11)}=\frac{\partial^{2} \mathrm{f}_{\mathrm{ij}}}{\partial \mathrm{v}_{\mathrm{i}}^{2}}, \\
& \mathrm{f}_{\mathrm{ij}}^{(12)}=\frac{\partial^{2} \mathrm{f}_{\mathrm{ij}}}{\partial \mathrm{v}_{\mathrm{i}} \mathrm{z}_{\mathrm{i}}}, \quad \mathrm{f}_{\mathrm{ij}}^{(22)}=\frac{\partial^{2} \mathrm{f}_{\mathrm{ij}}}{\partial \mathrm{z}_{\mathrm{i}}^{2}}, \\
& \mathrm{v}_{\mathrm{i}}^{\oplus}=1+\vartheta_{1}\left(\mathrm{v}_{\mathrm{i}}-1\right), \quad \mathrm{z}_{\mathrm{i}}^{\oplus}=1+\vartheta_{2}\left(\mathrm{z}_{\mathrm{i}}-1\right) ; \\
& 0<\vartheta_{1}, \vartheta_{2}<1 \forall i=1,2 .
\end{aligned}
$$

To obtain the bias and mean square error under large sample approximation up to order $\left(\mathrm{n}^{-1}\right)$, we assume

$$
\epsilon_{1}=\frac{\left(\overline{\mathrm{y}}_{1}^{*}-\overline{\mathrm{Y}}_{1}\right)}{\overline{\mathrm{Y}}_{1}}, \quad \in_{2}=\frac{\left(\overline{\mathrm{y}}_{2}^{*}-\overline{\mathrm{Y}}_{2}\right)}{\overline{\mathrm{Y}}_{2}}, \quad \in_{0}=\frac{\left(\overline{\mathrm{x}}^{*}-\overline{\mathrm{X}}\right)}{\overline{\mathrm{X}}}, \quad \in=\frac{\left(\bar{\phi}_{\mathrm{n}}^{*}-\bar{\emptyset}_{\mathrm{N}}\right)}{\bar{\emptyset}_{\mathrm{N}}}
$$

such that $\mathrm{E}\left(\epsilon_{1}\right)=0, \mathrm{E}\left(\epsilon_{2}\right)=0, \mathrm{E}\left(\epsilon_{0}\right)=0, \mathrm{E}(\epsilon)=0$.

Therefore

$$
\begin{aligned}
& \mathrm{E}\left(\in_{1}^{2}\right)=\frac{\mathrm{V}\left(\overline{\mathrm{y}}_{1}^{*}\right)}{\overline{\mathrm{Y}}_{1}^{2}}, \quad \mathrm{E}\left(\in_{2}^{2}\right)=\frac{\mathrm{V}\left(\overline{\mathrm{y}}_{2}^{*}\right)}{\overline{\mathrm{Y}}_{2}^{2}}, \quad \mathrm{E}\left(\in_{0}^{2}\right)=\frac{\mathrm{V}\left(\overline{\mathrm{x}}^{*}\right)}{\overline{\mathrm{X}}^{2}}, \\
& \mathrm{E}\left(\in^{2}\right)=\frac{\mathrm{v}\left(\bar{\phi}_{\mathrm{n}}^{*}\right)}{\bar{\emptyset}_{\mathrm{N}}^{2}}, \quad \mathrm{E}\left(\in_{1} \in_{2}\right)=\frac{\operatorname{Cov}\left(\overline{\mathrm{y}}_{1}^{*} \overline{\mathrm{Y}}_{2}^{*}\right)}{\overline{\mathrm{Y}}_{1} \overline{\mathrm{Y}}_{2}}, \\
& \mathrm{E}\left(\epsilon_{2} \in_{0}\right)=\frac{\operatorname{Cov}\left(\overline{\mathrm{y}}_{2}^{*}, \overline{\mathrm{x}}^{*}\right)}{\overline{\mathrm{Y}}_{2} \overline{\mathrm{X}}}, \quad \mathrm{E}\left(\epsilon_{1} \in_{0}\right)=\frac{\operatorname{Cov}\left(\overline{\mathrm{y}}_{1}^{*}, \overline{\mathrm{x}}^{*}\right)}{\overline{\mathrm{Y}}_{1} \overline{\mathrm{X}}}, \\
& \mathrm{E}\left(\in_{2} \in\right)=\frac{\operatorname{Cov}\left(\overline{\mathrm{y}}_{2}^{*}, \bar{\phi}_{\mathrm{n}}^{*}\right)}{\overline{\mathrm{Y}}_{2} \bar{\phi}_{\mathrm{N}}}, \quad \mathrm{E}\left(\in_{1} \in\right)=\frac{\operatorname{Cov}\left(\overline{\mathrm{y}}_{1}^{*}, \bar{\phi}_{\mathrm{n}}^{*}\right)}{\overline{\mathrm{Y}}_{1} \bar{\phi}_{\mathrm{N}}} \\
& \text { and } \quad \mathrm{E}\left(\in_{0} \in\right)=\frac{\operatorname{Cov}\left(\overline{\mathrm{x}}^{*}, \bar{\emptyset}_{\mathrm{n}}^{*}\right)}{\overline{\mathrm{X}}_{\mathrm{N}}}
\end{aligned}
$$

The expressions of the bias (B) and the mean square error (MSE) of $\mathrm{T}_{\mathrm{ij}}$ up to the first degree of approximation are given by

$$
\begin{gathered}
\mathrm{B}\left(\mathrm{T}_{11}\right)=\mathrm{B}\left(\mathrm{u}_{1}\right)+\theta_{1}\left[\mathrm{C}_{\mathrm{Rx}}^{*} \mathrm{f}_{11}^{(1)}+\mathrm{C}_{\mathrm{R} \emptyset}^{*} \mathrm{f}_{11}^{(2)}+\frac{1}{2}\left(\mathrm{~V}_{\mathrm{x}}^{*} \mathrm{f}_{11}^{(11)}+\right.\right. \\
\left.\left.2 \mathrm{~V}_{\mathrm{x} \emptyset}^{*} \mathrm{f}_{11}^{(12)}+\mathrm{V}_{\varnothing}^{*} \mathrm{f}_{11}^{(22)}\right)\right], \\
\mathrm{B}\left(\mathrm{T}_{21}\right)=\mathrm{B}\left(\mathrm{u}_{1}\right)+\mathrm{R}\left[\mathrm{C}_{\mathrm{Rx}} \mathrm{f}_{21}^{(1)}+\mathrm{C}_{\mathrm{R} \phi} \mathrm{f}_{21}^{(2)}+\frac{1}{2}\left(\mathrm{~V}_{\mathrm{x}} \mathrm{f}_{21}^{(11)}+\right.\right. \\
\left.\left.2 \mathrm{~V}_{\mathrm{x} \emptyset} \mathrm{f}_{21}^{(12)}+\mathrm{V}_{\emptyset} \mathrm{f}_{21}^{(22)}\right)\right], \\
\mathrm{B}\left(\mathrm{T}_{12}\right)=\mathrm{B}\left(\mathrm{u}_{2}\right)+\mathrm{P}\left[\mathrm{C}_{\mathrm{Px}}^{*} \mathrm{f}_{12}^{(1)}+\mathrm{C}_{\mathrm{P} \emptyset}^{*} \mathrm{f}_{12}^{(2)}+\frac{1}{2}\left(\mathrm{~V}_{\mathrm{x}}^{*} \mathrm{f}_{12}^{(11)}+\right.\right. \\
\left.\left.2 \mathrm{~V}_{\mathrm{x} \emptyset}^{*} \mathrm{f}_{12}^{(12)}+\mathrm{V}_{\varnothing}^{*} \mathrm{f}_{12}^{(22)}\right)\right], \\
\mathrm{B}\left(\mathrm{T}_{22}\right)=\mathrm{B}\left(\mathrm{u}_{2}\right)+\mathrm{P}\left[\mathrm{C}_{\mathrm{Px}} \mathrm{f}_{22}^{(1)}+\mathrm{C}_{\mathrm{P} \emptyset} \mathrm{f}_{22}^{(2)}+\frac{1}{2}\left(\mathrm{~V}_{\mathrm{x}} \mathrm{f}_{22}^{(11)}+\right.\right. \\
\left.\left.2 \mathrm{~V}_{\mathrm{x} \emptyset} \mathrm{f}_{22}^{(12)}+\mathrm{V}_{\varnothing} \mathrm{f}_{22}^{(22)}\right)\right],
\end{gathered}
$$

$\operatorname{MSE}\left(\mathrm{T}_{11}\right)=\left(\theta_{1}\right)^{2}\left[\mathrm{M}\left(\mathrm{u}_{1}\right)+\mathrm{V}_{\mathrm{x}}^{*}\left\{\mathrm{f}_{11}^{(1)}\right\}^{2}+\mathrm{V}_{\varnothing}^{*}\left\{\mathrm{f}_{11}^{(2)}\right\}^{2}+\right.$

$$
\left.2 \mathrm{C}_{\mathrm{x} \phi}^{*} \mathrm{f}_{11}^{(1)} \mathrm{f}_{11}^{(2)}+2 \mathrm{C}_{\mathrm{Rx}}^{*} \mathrm{f}_{11}^{(1)}+2 \mathrm{C}_{\mathrm{R} \phi}^{*} \mathrm{f}_{11}^{(2)}\right] \text {, }
$$

$\operatorname{MSE}\left(\mathrm{T}_{21}\right)=\left(\theta_{1}\right)^{2}\left[\mathrm{M}\left(\mathrm{u}_{1}\right)+\mathrm{V}_{\mathrm{x}}\left\{\mathrm{f}_{21}^{(1)}\right\}^{2}+\mathrm{V}_{\emptyset}\left\{\mathrm{f}_{21}^{(2)}\right\}^{2}+\right.$

$$
\left.2 \mathrm{C}_{\mathrm{x} \emptyset} \mathrm{f}_{21}^{(1)} \mathrm{f}_{21}^{(2)}+2 \mathrm{C}_{\mathrm{Rx}} \mathrm{f}_{21}^{(1)}+2 \mathrm{C}_{\mathrm{R} \emptyset} \mathrm{f}_{21}^{(2)}\right] \text {, }
$$

$\operatorname{MSE}\left(\mathrm{T}_{12}\right)=\left(\theta_{2}\right)^{2}\left[\mathrm{M}\left(\mathrm{u}_{2}\right)+\mathrm{V}_{\mathrm{x}}^{*}\left\{\mathrm{f}_{12}^{(1)}\right\}^{2}+\mathrm{V}_{\varnothing}^{*}\left\{\mathrm{f}_{12}^{(2)}\right\}^{2}+\right.$

$$
\left.2 \mathrm{C}_{\mathrm{x} \emptyset}^{*} \mathrm{f}_{12}^{(1)} \mathrm{f}_{12}^{(2)}+2 \mathrm{C}_{\mathrm{Rx}}^{*} \mathrm{f}_{12}^{(1)}+2 \mathrm{C}_{\mathrm{R} \emptyset}^{*} \mathrm{f}_{12}^{(2)}\right]
$$

and

$$
\begin{array}{r}
\operatorname{MSE}\left(T_{22}\right)=\left(\theta_{2}\right)^{2}\left[M\left(u_{2}\right)+V_{x}\left\{f_{22}^{(1)}\right\}^{2}+V_{\emptyset}\left\{f_{22}^{(2)}\right\}^{2}+\right. \\
\left.2 C_{x \emptyset} f_{22}^{(1)} f_{22}^{(2)}+2 C_{R x} f_{22}^{(1)}+2 C_{R \varnothing} f_{22}^{(2)}\right] .
\end{array}
$$

To obtain the minimum mean square error of $\mathrm{T}_{\mathrm{ij}}(\mathrm{i}=1,2 ; \mathrm{j}=$ 1,2 ), we differentiate equation (36) partially with respect to $v_{1}, z_{1}$ and equating them to zero we have

$$
\frac{\partial \mathrm{T}_{11}}{\partial \mathrm{v}_{1}}=\mathrm{V}_{\mathrm{x}}^{*} \mathrm{f}_{11}^{(1)}+\mathrm{C}_{\mathrm{x} \emptyset}^{*} \mathrm{f}_{11}^{(2)}+\mathrm{C}_{\mathrm{Rx}}^{*}=0
$$

and

$$
\frac{\partial \mathrm{T}_{11}}{\partial \mathrm{z}_{1}}=\mathrm{V}_{\varnothing}^{*} \mathrm{f}_{11}^{(2)}+\mathrm{C}_{\mathrm{x} \emptyset}^{*} \mathrm{f}_{11}^{(1)}+\mathrm{C}_{\mathrm{R} \emptyset}^{*}=0 .
$$


Now solving the equations (40) and (41), we find

$$
\mathrm{f}_{11}^{(1)}=\frac{\mathrm{C}_{\mathrm{R} \emptyset}^{*} \mathrm{C}_{\mathrm{x} \emptyset}^{*}-\mathrm{V}_{\varnothing}^{*} \mathrm{C}_{\mathrm{Rx}}^{*}}{\mathrm{~V}_{\varnothing}^{*} \mathrm{~V}_{\mathrm{x}}^{*}-\left[\mathrm{C}_{\mathrm{x} \emptyset}^{*}\right]^{2}}
$$

and

$$
\mathrm{f}_{11}^{(2)}=-\frac{\mathrm{C}_{\mathrm{R} \emptyset}^{*}}{\mathrm{~V}_{\emptyset}^{*}}-\frac{\mathrm{C}_{\mathrm{x} \emptyset}^{*}\left(\mathrm{C}_{\mathrm{R} \emptyset}^{*} \mathrm{C}_{\mathrm{x} \emptyset}^{*}-\mathrm{V}_{\emptyset}^{*} \mathrm{C}_{\mathrm{Rx}}^{*}\right)}{\mathrm{V}_{\emptyset}^{*}\left(\mathrm{~V}_{\varnothing}^{*} \mathrm{~V}_{\mathrm{x}}^{*}-\left[\mathrm{C}_{\mathrm{x} \emptyset}^{*}\right]^{2}\right)}
$$

It may be easily verified that $\mathrm{f}_{11}^{(1)}$ and $\mathrm{f}_{11}^{(2)}$ minimize the value of MSE $\left(\mathrm{T}_{11}\right)$. So, the mean square error of the proposed families of estimators $\mathrm{T}_{11}$ will be minimum if $\mathrm{f}_{11}^{(1)}$ and $\mathrm{f}_{11}^{(2)}$ are calculated by the equations (42) and (43).

The minimum mean square error of $\mathrm{T}_{11}$ using $\mathrm{f}_{11}^{(1)}$ and $\mathrm{f}_{11}^{(2)}$ respectively from the equations (42) and (43) is as follows

$\operatorname{MSE}\left(\mathrm{T}_{11}\right)_{\min }=\left(\theta_{1}\right)^{2}\left[\mathrm{M}\left(\mathrm{u}_{1}\right)-\frac{\left[\mathrm{C}_{\mathrm{R} \phi}^{*}\right]^{2}}{\mathrm{~V}_{\emptyset}^{*}}-\frac{\left(\mathrm{C}_{\mathrm{R} \emptyset}^{*} \mathrm{C}_{\mathrm{x} \emptyset}^{*}-\mathrm{V}_{\varnothing}^{*} \mathrm{C}_{\mathrm{Rx}}^{*}\right)^{2}}{\mathrm{~V}_{\varnothing}^{*}\left(\mathrm{~V}_{\varnothing}^{*} \mathrm{~V}_{\mathrm{x}}^{*}-\left[\mathrm{C}_{\mathrm{x} \phi}^{*}\right]^{2}\right)}\right]$

where,

$\mathrm{C}_{\mathrm{x} \emptyset}^{*}=\frac{\mathrm{N}-\mathrm{n}}{\mathrm{Nn}}\left(\rho_{30} \mathrm{C}_{\mathrm{x}} \mathrm{C}_{\varnothing}\right)+\frac{\mathrm{w}_{2}(\mathrm{k}-1)}{\mathrm{n}}\left(\rho_{30}^{\prime} \mathrm{C}_{\mathrm{x}}^{\prime} \mathrm{C}_{\emptyset}^{\prime}\right)$

and

$$
\begin{aligned}
C_{R x}^{*}=\frac{N-n}{N n}\left(\rho_{13} C_{y_{1}}-\rho_{23} C_{y_{2}}\right) C_{x} & \\
& +\frac{\mathrm{w}_{2}(k-1)}{n}\left(\rho_{13}^{\prime} C_{y_{1}}^{\prime}-\rho_{23}^{\prime} C_{y_{2}}^{\prime}\right) C_{x}^{\prime}
\end{aligned}
$$

Similarly, the conditions for which the families of estimators $\mathrm{T}_{21}, \mathrm{~T}_{12}$ and $\mathrm{T}_{22}$ attain their minimum mean square error can be obtained by differentiating equations (37), (38) and (39) with respect to the corresponding $\mathrm{v}_{\mathrm{i}}, \mathrm{z}_{\mathrm{i}}(\mathrm{i}=1,2 ; \mathrm{j}=1,2)$ and equating to zero. After solving them we have:

i) Conditions for minimum mean square error of $\mathrm{T}_{21}$

$$
f_{21}^{(1)}=\frac{C_{R \varnothing} C_{x \emptyset}-V_{\varnothing} C_{R x}}{V_{\varnothing} V_{x}-C_{x \emptyset}^{2}}
$$

and

$$
\mathrm{f}_{21}^{(2)}=-\frac{\mathrm{C}_{\mathrm{R} \emptyset}}{\mathrm{V}_{\varnothing}}-\frac{\mathrm{C}_{\mathrm{x} \phi}\left(\mathrm{C}_{\mathrm{R} \phi} \mathrm{C}_{\mathrm{x} \emptyset}-\mathrm{V}_{\varnothing} \mathrm{C}_{\mathrm{Rx}}\right)}{\mathrm{V}_{\varnothing}\left(\mathrm{V}_{\varnothing} \mathrm{V}_{\mathrm{x}}-\mathrm{C}_{\mathrm{x} \emptyset}^{2}\right)}
$$

ii) Conditions for minimum mean square error of $\mathrm{T}_{12}$

$$
f_{12}^{(1)}=\frac{C_{P \emptyset}^{*} C_{x \emptyset}^{*}-V_{\varnothing}^{*} C_{P x}^{*}}{V_{\emptyset}^{*} V_{x}^{*}-\left[C_{x \emptyset}^{*}\right]^{2}}
$$

and

$$
\mathrm{f}_{12}^{(2)}=-\frac{\mathrm{C}_{\mathrm{P} \emptyset}^{*}}{\mathrm{~V}_{\varnothing}^{*}}-\frac{\mathrm{C}_{\mathrm{x} \emptyset}^{*}\left(\mathrm{C}_{\mathrm{P} \emptyset}^{*} \mathrm{C}_{\mathrm{x} \emptyset}^{*}-\mathrm{V}_{\emptyset}^{*} \mathrm{C}_{\mathrm{Px}}^{*}\right)}{\mathrm{V}_{\emptyset}^{*}\left(\mathrm{~V}_{\varnothing}^{*} \mathrm{~V}_{\mathrm{x}}^{*}-\left[\mathrm{C}_{\mathrm{x} \emptyset}^{*}\right]^{2}\right)}
$$

iii) Conditions for minimum mean square error of $\mathrm{T}_{22}$

$$
\mathrm{f}_{22}^{(1)}=\frac{\mathrm{C}_{\mathrm{P} \emptyset} \mathrm{C}_{\mathrm{x} \emptyset}-\mathrm{V}_{\varnothing} \mathrm{C}_{\mathrm{Px}}}{\mathrm{V}_{\varnothing} \mathrm{V}_{\mathrm{x}}-\mathrm{C}_{\mathrm{x} \emptyset}^{2}}
$$

and

$$
\mathrm{f}_{22}^{(2)}=-\frac{\mathrm{C}_{\mathrm{P} \emptyset}}{\mathrm{V}_{\varnothing}}-\frac{\mathrm{C}_{\mathrm{x} \emptyset}\left(\mathrm{C}_{\mathrm{P} \emptyset} \mathrm{C}_{\mathrm{x} \emptyset}-\mathrm{V}_{\varnothing} \mathrm{C}_{\mathrm{Px}}\right)}{\mathrm{V}_{\emptyset}\left(\mathrm{V}_{\varnothing} \mathrm{V}_{\mathrm{x}}-\mathrm{C}_{\mathrm{x} \emptyset}^{2}\right)} .
$$

Now putting the value of $\mathrm{f}_{21}^{(1)}$ and $\mathrm{f}_{21}^{(2)}$ in equation $(37), \mathrm{f}_{12}^{(1)}$ and $\mathrm{f}_{12}^{(2)}$ in equation (38), $\mathrm{f}_{21}^{(1)}$ and $\mathrm{f}_{21}^{(2)}$ in equation (39), the minimum mean square errors of $\mathrm{T}_{21}, \mathrm{~T}_{12}$ and $\mathrm{T}_{22}$ are as follows

$$
\begin{aligned}
& \operatorname{MSE}\left(\mathrm{T}_{21}\right)_{\min }=\left(\theta_{1}\right)^{2}\left[\mathrm{M}\left(\mathrm{u}_{1}\right)-\frac{\left[\mathrm{C}_{\mathrm{R} \emptyset}\right]^{2}}{\mathrm{~V}_{\emptyset}}-\frac{\left(\mathrm{C}_{\mathrm{R} \phi} \mathrm{C}_{\mathrm{x} \emptyset}-\mathrm{V}_{\emptyset} \mathrm{C}_{\mathrm{Rx}}\right)^{2}}{\mathrm{~V}_{\emptyset}\left(\mathrm{V}_{\varnothing} \mathrm{V}_{\mathrm{x}}-\mathrm{C}_{\mathrm{x} \emptyset}^{2}\right)}\right], \\
& \operatorname{MSE}\left(\mathrm{T}_{12}\right)_{\min }=\left(\theta_{2}\right)^{2}\left[\mathrm{M}\left(\mathrm{u}_{2}\right)-\frac{\left[\mathrm{C}_{\mathrm{P} \phi}^{*}\right]^{2}}{\mathrm{~V}_{\varnothing}^{*}}-\frac{\left(\mathrm{C}_{\mathrm{P} \varnothing}^{*} \mathrm{C}_{\mathrm{x} \emptyset}^{*}-\mathrm{V}_{\varnothing}^{*} \mathrm{C}_{\mathrm{Px}}^{*}\right)^{2}}{\mathrm{~V}_{\varnothing}^{*}\left(\mathrm{~V}_{\varnothing}^{*} \mathrm{~V}_{\mathrm{x}}^{*}-\left[\mathrm{C}_{\mathrm{x} \varnothing}^{*}\right]^{2}\right)}\right]
\end{aligned}
$$

and

$\operatorname{MSE}\left(\mathrm{T}_{22}\right)_{\min }=\left(\theta_{2}\right)^{2}\left[\mathrm{M}\left(\mathrm{u}_{2}\right)-\frac{\left[\mathrm{C}_{\mathrm{P} \emptyset}\right]^{2}}{\mathrm{~V}_{\varnothing}}-\frac{\left(\mathrm{C}_{\mathrm{P} \phi} \mathrm{C}_{\mathrm{x} \emptyset}-\mathrm{V}_{\emptyset} \mathrm{C}_{\mathrm{Px}}\right)^{2}}{\mathrm{~V}_{\emptyset}\left(\mathrm{V}_{\varnothing} \mathrm{V}_{\mathrm{x}}-\mathrm{C}_{\mathrm{x} \emptyset}^{2}\right)}\right]$

The proposed families of estimators are the function of sample values and many types of functions may be possible under the discussed condition. So some members of these families of estimators $\mathrm{T}_{\mathrm{ij}}$ may be considered as

$$
\begin{aligned}
& T_{i j}(i j)=u_{j} v_{i}{ }^{a_{i j}} z_{i} b_{i j}, \quad(i=1,2 ; j=1,2)
\end{aligned}
$$

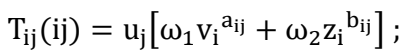

$$
\begin{aligned}
& \omega_{1}+\omega_{2}=1, \quad(i=1,2 ; j=1,2) \\
& \mathrm{T}_{\mathrm{ij}}(\mathrm{ij})=\mathrm{u}_{\mathrm{j}}\left[\omega_{1}\left(2-\mathrm{v}_{\mathrm{i}}\right)+\omega_{2}\left(2-\mathrm{z}_{\mathrm{i}}\right)\right] \text {; } \\
& \omega_{1}+\omega_{2}=1, \quad(i=1,2 ; j=1,2)
\end{aligned}
$$

Sometimes the values of parameters in the optimum values of the constants are not known then one may estimate them on the basis of the sample values or may use past data. (Reddy 1978) has shown that such values are not only stable overtime and region but also don't affect the mean square error of the estimators up to the terms of order $n^{-1}$ (Srivastava \& Jhaji 1983).

\section{Comparisons of efficiency of proposed class of estimators with relevant estimators}

When we compare the efficiency of the proposed estimators $\mathrm{T}_{\mathrm{ij}}=\mathrm{u}_{\mathrm{j}} \mathrm{f}_{\mathrm{ij}}\left(\mathrm{v}_{\mathrm{i}}, \mathrm{z}_{\mathrm{i}}\right), \mathrm{i}=1,2 ; \mathrm{j}=1,2$, with relevant estimators, we observe

i) From equation (10) and (18)

$$
\operatorname{MSE}\left(\mathrm{u}_{1}\right)-\operatorname{MSE}\left(\mathrm{t}_{11}\right)_{\min }=\left(\theta_{1}\right)^{2} \frac{\left[\mathrm{C}_{\mathrm{Rx}}^{*}\right]^{2}}{\mathrm{~V}_{\mathrm{x}}^{*}}>0
$$

ii) From equation (11) and (18)

$$
\operatorname{MSE}\left(\mathrm{u}_{1}\right)-\operatorname{MSE}\left(\mathrm{t}_{12}\right)_{\min }=\left(\theta_{1}\right)^{2} \frac{\left[\mathrm{C}_{\mathrm{Rx}}\right]^{2}}{\mathrm{~V}_{\mathrm{x}}}>0
$$

iii) From equation (12) and (19)

$$
\operatorname{MSE}\left(\mathrm{u}_{2}\right)-\operatorname{MSE}\left(\mathrm{t}_{21}\right)_{\min }=\left(\theta_{2}\right)^{2} \frac{\left[\mathrm{C}_{\mathrm{Px}}^{*}\right]^{2}}{\mathrm{~V}_{\mathrm{x}}^{*}}>0
$$

iv) From equation (13) and (19)

$$
\operatorname{MSE}\left(u_{2}\right)-\operatorname{MSE}\left(t_{22}\right)_{\min }=\left(\theta_{2}\right)^{2} \frac{\left[\mathrm{C}_{\mathrm{Px}}\right]^{2}}{\mathrm{~V}_{\mathrm{x}}}>0
$$

v) From equation (14) and (18)

$$
\operatorname{MSE}\left(\mathrm{u}_{1}\right)-\operatorname{MSE}\left(\mathrm{t}_{31}\right)_{\min }=\left(\theta_{1}\right)^{2} \frac{\left[\mathrm{C}_{\mathrm{R} \emptyset}^{*}\right]^{2}}{\mathrm{~V}_{\emptyset}^{*}}>0
$$

vi) From equation (15) and (18)

$$
\operatorname{MSE}\left(\mathrm{u}_{1}\right)-\operatorname{MSE}\left(\mathrm{t}_{32}\right)_{\min }=\left(\theta_{1}\right)^{2} \frac{\left[\mathrm{C}_{\mathrm{R} \emptyset}\right]^{2}}{\mathrm{~V}_{\emptyset}}>0
$$


vii) From equation (16) and (19)

$$
\operatorname{MSE}\left(\mathrm{u}_{2}\right)-\operatorname{MSE}\left(\mathrm{t}_{41}\right)_{\min }=\left(\theta_{2}\right)^{2} \frac{\left[\mathrm{C}_{\mathrm{P} \emptyset}^{*}\right]^{2}}{\mathrm{~V}_{\emptyset}^{*}}>0
$$

viii) From equation (17) and (19)

$$
\operatorname{MSE}\left(u_{2}\right)-\operatorname{MSE}\left(t_{42}\right)_{\min }=\left(\theta_{2}\right)^{2} \frac{\left[\mathrm{C}_{P \emptyset}\right]^{2}}{V_{\varnothing}}>0
$$

ix) From equation (18) and (44)

$$
\begin{aligned}
& \operatorname{MSE}\left(\mathrm{u}_{1}\right)-\operatorname{MSE}\left(\mathrm{T}_{11}\right)_{\min } \\
& =\left(\theta_{1}\right)^{2}\left[\frac{\left[\mathrm{C}_{\mathrm{R} \emptyset}^{*}\right]^{2}}{\mathrm{~V}_{\varnothing}^{*}}+\frac{\left(\mathrm{C}_{\mathrm{R} \emptyset}^{*} \mathrm{C}_{\mathrm{x} \emptyset}^{*}-\mathrm{V}_{\varnothing}^{*} \mathrm{C}_{\mathrm{Rx}}^{*}\right)^{2}}{\mathrm{~V}_{\varnothing}^{*}\left(\mathrm{~V}_{\varnothing}^{*} \mathrm{~V}_{\mathrm{x}}^{*}-\left[\mathrm{C}_{\mathrm{x} \varnothing}^{*}\right]^{2}\right)}\right]>0
\end{aligned}
$$

x) From equation (18) and (53)

$$
\begin{array}{r}
\operatorname{MSE}\left(u_{1}\right)-\operatorname{MSE}\left(T_{21}\right)_{\min } \\
=\left(\theta_{1}\right)^{2}\left[\frac{\left[C_{R \varnothing}\right]^{2}}{V_{\varnothing}}+\frac{\left(C_{R \varnothing} C_{x \emptyset}-V_{\varnothing} C_{R x}\right)^{2}}{V_{\varnothing}\left(V_{\varnothing} V_{x}-C_{x \emptyset}^{2}\right)}\right]>0
\end{array}
$$

xi) From equation (19) and (54)

$$
\begin{aligned}
\operatorname{MSE}\left(\mathrm{u}_{2}\right)-\operatorname{MSE}\left(\mathrm{T}_{12}\right)_{\min } & \\
& =\left(\theta_{2}\right)^{2}\left[\frac{\left[\mathrm{C}_{\mathrm{P} \emptyset}^{*}\right]^{2}}{\mathrm{~V}_{\varnothing}^{*}}+\frac{\left(\mathrm{C}_{\mathrm{P} \emptyset}^{*} \mathrm{C}_{\mathrm{x} \emptyset}^{*}-\mathrm{V}_{\varnothing}^{*} \mathrm{C}_{\mathrm{Px}}^{*}\right)^{2}}{\mathrm{~V}_{\varnothing}^{*}\left(\mathrm{~V}_{\varnothing}^{*} \mathrm{~V}_{\mathrm{x}}^{*}-\left[\mathrm{C}_{\mathrm{x} \varnothing}^{*}\right]^{2}\right)}\right]>0
\end{aligned}
$$

xii) From equation (19) and (55)

$\operatorname{MSE}\left(\mathrm{u}_{2}\right)-\operatorname{MSE}\left(\mathrm{T}_{22}\right)_{\min }$

$$
=\left(\theta_{2}\right)^{2}\left[\frac{\left[\mathrm{C}_{\mathrm{P} \emptyset}\right]^{2}}{\mathrm{~V}_{\varnothing}}+\frac{\left(\mathrm{C}_{\mathrm{P} \emptyset} \mathrm{C}_{\mathrm{x} \emptyset}-\mathrm{V}_{\varnothing} \mathrm{C}_{\mathrm{Px}}\right)^{2}}{\mathrm{~V}_{\varnothing}\left(\mathrm{V}_{\varnothing} \mathrm{V}_{\mathrm{x}}-\mathrm{C}_{\mathrm{x} \emptyset}^{2}\right)}\right]>0
$$

xiii) From equation (14), (18) and (44)

$$
\operatorname{MSE}\left(\mathrm{T}_{11}\right)_{\min }<\operatorname{MSE}\left(\mathrm{t}_{31}\right)_{\min }<M S E\left(\mathrm{u}_{1}\right)
$$

xiv) From equation (15), (18) and (53)

$$
\operatorname{MSE}\left(\mathrm{T}_{21}\right)_{\min }<\operatorname{MSE}\left(\mathrm{t}_{32}\right)_{\min }<\operatorname{MSE}\left(\mathrm{u}_{1}\right)
$$

xv) From equation (16), (19) and (54)

$$
\operatorname{MSE}\left(\mathrm{T}_{12}\right)_{\min }<M S E\left(\mathrm{t}_{41}\right)_{\min }<M S E\left(\mathrm{u}_{2}\right)
$$

xvi) From equation (17), (19) and (55)

$$
\operatorname{MSE}\left(\mathrm{T}_{22}\right)_{\min }<\operatorname{MSE}\left(\mathrm{t}_{42}\right)_{\min }<M S E\left(\mathrm{u}_{2}\right)
$$

\section{An empirical study}

\section{Data Set-I}

96 village wise population of rural area under Police-station Singur, District - Hooghly, West Bengal has been taken under the study from District Census Handbook 1981 published by Govt. of India. The last $25 \%$ villages (i.e. 24 villages) have been considered as non-responding group of the population. Here we have taken the study characters and auxiliary characters as follows:

$\mathrm{y}_{1}$ : Number of agricultural laborers in the village.

$\mathrm{y}_{2}$ : Total area of the village.

$\mathrm{x}:$ Number of occupied houses in the village.

$\emptyset$ : Number of occupied houses less than or equal to 250 .

The parameters of this data set are as follows:

\begin{tabular}{lllll}
\hline \multirow{2}{*}{$\mathrm{N}=96$} & $\begin{array}{l}\overline{\mathrm{Y}}_{1} \\
=162.67\end{array}$ & $\begin{array}{l}\overline{\mathrm{Y}}_{2} \\
=164.01\end{array}$ & $\begin{array}{l}\overline{\mathrm{X}} \\
=303.10\end{array}$ & $\begin{array}{l}\bar{\phi} \\
=0.6146\end{array}$ \\
\hline $\mathrm{C}_{\mathrm{y}_{1}}$ & $\mathrm{C}_{\mathrm{y}_{2}}$ & $\mathrm{C}_{\mathrm{x}}$ & $\mathrm{C}_{\phi}$ & $\rho$ \\
$=1.431$ & $=0.800$ & $=1.163$ & $=0.796$ & $=0.779$ \\
$\rho_{13}$ & $\rho_{10}$ & $\rho_{23}$ & $\rho_{20}$ & $\rho_{30}$ \\
$=0.915$ & $=0.502$ & $=0.886$ & $=0.643$ & $=0.629$ \\
$\mathrm{~N}_{2}=24$ & $\overline{\mathrm{Y}}_{1(2)}$ & $\overline{\mathrm{Y}}_{2(2)}$ & $\overline{\mathrm{X}}_{(2)}$ & $\bar{\phi}_{(2)}$ \\
& $=216.62$ & $=183.32$ & $=361.96$ & $=0.5833$ \\
$\mathrm{C}_{\mathrm{y}_{1}}^{\prime}$ & $\mathrm{C}_{\mathrm{y}_{2}}^{\prime}$ & $\mathrm{C}_{\mathrm{x}}^{\prime}$ & $\mathrm{C}_{\phi}^{\prime}$ & $\rho_{(2)}$ \\
$=1.859$ & $=0.857$ & $=1.402$ & $=0.982025$ & $=0.791$ \\
$\rho_{13}^{\prime}$ & $\rho_{10}^{\prime}$ & $\rho_{23}^{\prime}$ & $\rho_{20}^{\prime}$ & $\rho_{30}^{\prime}$ \\
$=0.926$ & $=0.489$ & $=0.883$ & $=0.660$ & $=0.608$ \\
\hline & & & &
\end{tabular}

We have considered Data set $I$ to estimate $\theta_{1}$ and the tors $\mathrm{T}_{11}(11)=\mathrm{u}_{1} \mathrm{v}_{1}{ }^{\mathrm{a}_{11}} \mathrm{z}_{1} \mathrm{~b}_{11}$ and $\mathrm{T}_{21}(21)=\mathrm{u}_{1} \mathrm{v}_{2}{ }^{\mathrm{a}_{21}} \mathrm{z}_{2}{ }^{\mathrm{b}_{21}}$ have been considered for comparing the efficiency of the proposed families of estimators $\mathrm{T}_{11}$ and $\mathrm{T}_{21}$ with other relevant estimators which is shown in Table 1.

\section{Data Set II}

109 Village/Town/ward wise population of urban area under Police Station -Baria, Tahasil-Champua, Orissa, India has been taken under the study from District Census Handbook, 1981, Orissa, published by Govt. of India. The first $25 \%$ villages (i.e. 27 villages) have been considered as non-response group of the population. Here we have taken the study characters and auxiliary characters as follows:

$\mathrm{y}_{1}$ : Number of occupied houses in the village.

$\mathrm{y}_{2}$ : Average number of persons in the village.

$\mathrm{x}$ : Total area of the village.

$\varnothing$ : Area greater than equal to 150

The parameters of the population are as follows

\begin{tabular}{lllll}
\hline $\mathrm{N}=109$ & $\begin{array}{l}\overline{\mathrm{Y}}_{1} \\
=88.8624\end{array}$ & $\begin{array}{l}\overline{\mathrm{Y}}_{2} \\
=5.5547\end{array}$ & $\begin{array}{l}\overline{\mathrm{X}} \\
=255.97\end{array}$ & $\begin{array}{l}\bar{\phi} \\
=0.7248\end{array}$ \\
\hline $\mathrm{C}_{\mathrm{y}_{1}}$ & $\mathrm{C}_{\mathrm{y}_{2}}$ & $\mathrm{C}_{\mathrm{x}}$ & $\mathrm{C}_{\phi}$ & $\mathrm{C}_{\mathrm{y}_{1}}^{\prime}$ \\
$=0.6639$ & $=0.1263$ & $=0.6065$ & $=0.6191$ & $=0.7660$ \\
$\mathrm{C}_{\mathrm{y}_{2}}^{\prime}$ & $\mathrm{C}_{\mathrm{x}}^{\prime}$ & $\mathrm{C}_{\phi}^{\prime}$ & $\rho$ & $\rho_{13}$ \\
$=0.1052$ & $=0.7029$ & $=0.4994$ & $=-0.188$ & $=0.851$ \\
$\rho_{10}$ & $\rho_{23}$ & $\rho_{20}$ & $\rho_{30}$ & $\rho_{(2)}$ \\
$=0.492$ & $=-0.082$ & $=-0.068$ & $=0.592$ & $=-0.074$ \\
$\rho_{13}^{\prime}$ & $\rho_{10}^{\prime}$ & $\rho_{23}^{\prime}$ & $\rho_{20}^{\prime}$ & $\rho_{30}^{\prime}$ \\
$=0.806$ & $=0.316$ & $=0.078$ & $=0.028$ & $=0.028$ \\
\hline
\end{tabular}

Data Set II is considered to estimate $\theta_{2}$ and to show the efficiency of the proposed families of estimators over the relevant estimators, $\mathrm{T}_{12}(12)=\mathrm{u}_{2} \mathrm{v}_{1}{ }^{\mathrm{a}_{12}} \mathrm{z}_{1} \mathrm{~b}_{12}$ and $\mathrm{T}_{22}(22)=\mathrm{u}_{2} \mathrm{v}_{2}{ }^{\mathrm{a}_{22}} \mathrm{z}_{2}{ }^{\mathrm{b}_{22}}$ have been considered to calculate the relative efficiency of the proposed families of estimators $T_{12}$ and $T_{22}$ with respect to other relevant estimators, which is shown in Table 2 .

The relative efficiency (R.E.) of the estimators in percentage is calculated by the formula

R. E. $=\frac{\operatorname{MSE}(\widehat{\mathrm{R}})}{\operatorname{MSE}(.)} \times 100$.

Table 1: R. E. (.) (in \%) of estimator with respect to $\mathrm{u}_{1}$ for different values of $\mathrm{k}$

\begin{tabular}{cccc}
\hline \multirow{2}{*}{ Estimators } & & $(\mathrm{N}=96, \mathrm{n}=30)$ \\
& & $1 / \mathrm{k}$ & \\
\hline \multirow{2}{*}{$\mathrm{u}_{1}$} & $1 / 4$ & $1 / 3$ & $1 / 2$ \\
& $(61394.20)^{*}$ & 100.00 & 100.00 \\
$\mathrm{t}_{31}$ & 106.28 & $(46618.38)$ & $(33485.89)$ \\
& $(57762.15)$ & $(44258.22)$ & $(31724.33)$ \\
$\mathrm{T}_{11}$ & 172.00 & 158.00 & 141.60 \\
& $(35687.18)$ & $(29471.33)$ & $(23636.92)$ \\
$\mathrm{t}_{32}$ & 101.54 & 102.04 & 102.87 \\
& $(60457.75)$ & $(45681.93)$ & $(32549.44)$ \\
$\mathrm{T}_{21}$ & 105.80 & 107.79 & 111.19 \\
& $(58024.20)$ & $(43248.40)$ & $(30115.90)$ \\
\hline
\end{tabular}

* Figures in parenthesis give MSE(.) in $10^{-6}$. 
Table 2: R. E. (.) (in \%) of estimators with respect to $\mathrm{u}_{2}$ for different values of $\mathrm{k}$

\begin{tabular}{cccc}
\hline Estimators & \multicolumn{3}{c}{$(\mathrm{N}=109, \mathrm{n}=30)$} \\
$1 / \mathrm{k}$ & \\
\hline & $1 / 4$ & $1 / 3$ & $1 / 2$ \\
$\mathrm{u}_{2}$ & 100.00 & 100.00 & 100.00 \\
& $(6091.12)^{*}$ & $(4872.90)$ & $(3703.40)$ \\
$\mathrm{t}_{41}$ & 118.62 & 120.85 & 124.36 \\
& $(5134.78)$ & $(4032.14)$ & $(2978.07)$ \\
$\mathrm{T}_{12}$ & 324.12 & 327.17 & 339.35 \\
& $(1871.87)$ & $(1489.65)$ & $(1091.32)$ \\
$\mathrm{t}_{42}$ & 110.82 & 113.91 & 119.14 \\
& $(5496.25)$ & $(4278.02)$ & $(3108.53)$ \\
$\mathrm{T}_{22}$ & 134.89 & 147.77 & 174.03 \\
& $(4515.79)$ & $(3297.56)$ & $(2128.07)$ \\
\hline
\end{tabular}

* Figures in parenthesis give MSE( $\cdot)$

\section{Conclusion}

From Table 1, it has been observed that the estimators $\mathrm{T}_{11}$ and $\mathrm{T}_{21}$ are more efficient than the corresponding estimators $t_{31}, t_{32}$ and conventional estimator $\mathrm{u}_{1}$ at different level of sub sampling fraction $(1 / \mathrm{k})$. The mean square error of $\mathrm{T}_{11}$ and $\mathrm{T}_{21}$ decreases as the sub-sampling fraction increases but on comparing the relative efficiency (R.E.) of $T_{11}$ and $T_{21}$ with respect to $u_{1}$, we observe that the R.E. of $\left(\mathrm{T}_{11}\right)$ with respect to $u_{1}$ decreases but the R. E. of $\left(\mathrm{T}_{21}\right)$ with respect to $\mathrm{u}_{1}$ increases as sub-sampling fraction increases. This is due to the fact that $\operatorname{MSE}\left(\mathrm{u}_{1}\right)$ decreases at a faster rate than $\operatorname{MSE}\left(\mathrm{T}_{11}\right)$ as sub-sampling fraction increases.

Similarly, Table 2 shows that the estimators $\mathrm{T}_{12}$ and $\mathrm{T}_{22}$ are more efficient than $t_{41}, t_{42}$ and conventional estimator $u_{2}$ at different level of sub sampling fraction $(1 / \mathrm{k})$. On comparing the relative efficiency (R.E.), we observe that R. E. of all the estimators $t_{41}$, $t_{42}, T_{12}$ and $T_{22}$ with respect to $u_{2}$ increases while their mean square errors decrease as sub-sampling fraction increases.

\section{References}

[1] Hansen MH \& Hurwitz WN (1946) the problems of non-response in sample surveys. Journal of American Statistical Association 41 517-529.http://dx.doi.org/10.1080/01621459.1946.10501894.

[2] Jhajj HS, Sharma MK \& Grover LK (2006) a family of estimators of population mean using information on auxiliary attribute. Pakistan Journal of Statistics 22, 43-50.

[3] Khare BB (1987) on modified class of estimators of ratio and product of two populations means using auxiliary character. Proc Math Soc BHU 3, 131-137.

[4] Khare BB (1990) a generalized class of estimators for a combination of products and ratio of some population means using multiauxiliary characters. J Stat Res 24(1-2), 1-8.

[5] Khare BB (1991) on generalized class of estimators for ratio of two populations means using multi-auxiliary characters. Aligarh J Stat $11,81-90$.

[6] Khare BB \& Pandey SK (2000) a class of estimators for ratio of two population means using auxiliary character in presence of nonresponse. J Sc Res BHU 50, 115-124.

[7] Khare BB \& Sinha RR (2002) Estimation of the ratio of two populations means using auxiliary character with unknown population means in presence of non-response, Prog of Maths BHU 36, 337 348.

[8] Khare BB \& Sinha RR (2004) Estimation of finite population ratio using two phase 8sampling in presence of non-response. Aligarh $\mathrm{J}$ Stat 24, 43-56.

[9] Khare BB \& Sinha RR (2007) Estimation of the ratio of the two populations means using multi-auxiliary characters in the presence of non-response. Statistical Techniques in Life Testing, Reliability, Sampling Theory and Quality Control, edited by B. N. Pandey, Narosa Publishing House, New House, New Delhi, 163-171.

[10] Khare BB \& Sinha RR (2012 a) Combined class of estimators for ratio and product of two population means in presence of nonresponse. International Journal of Statistics and Economics 8, 86-95.

[11] Khare BB \& Sinha RR (2012 b) Improved classes of estimators for ratio of two means with double sampling the non-respondents. Statistika 49(3), 75-83
[12] Koyuncu N (2012) efficient estimators of population mean using auxiliary attributes. Applied Mathematics and Computation 218 , 10900-10905.http://dx.doi.org/10.1016/j.amc.2012.04.050.

[13] Naik VD \& Gupta PC (1996) A note on estimating of mean with known population of an auxiliary character. Journal of Indian Society of Agricultural Statistics 48, 151-158.

[14] Rao PSRS (1986) Ratio estimation with subsampling the nonrespondents. Survey Methodology 12(2), 217-230.

[15] Reddy VN (1978) A study of use of prior knowledge on certain population parameters in estimation, Sankhya 40, 29-37.

[16] Shabbir J \& Gupta S (2010) Estimation of the finite population mean in two phase sampling when auxiliary variables are attributes. Hacettepe Journal of Mathematics and Statistics 39, 121-129.

[17] Shah SM \& Shah DN (1978) Ratio cum product estimator for estimating ratio (product) of two population parameters. Sankhya 40, 156-166.

[18] Singh HP (1965) on the estimation of ratio and product of two finite population means. Sankhya 27, 321-328.

[19] Singh HP \& Solanki RS (2012) Improved estimation of population mean in simple random sampling using information on auxiliary attribute. Applied Mathematics and computation 218, 7798 7812.http://dx.doi.org/10.1016/j.amc.2012.01.047.

[20] Singh RK (1982a) Generalized estimators for the estimation of ratio and product of population parameters. J Statist Res 16, 15-23.

[21] Singh RK (1982b) on estimating ratio and product of population parameters. Calcutta Statist Assoc 20, 39-49.

[22] Singh, R, Chauhan P, Sawan N \& Smarandache F (2008) Ratio estimators in simple random sampling using information on auxiliary attribute. Pakistan Journal of Statistics and Operations Research 4 47-53. http://dx.doi.org/10.18187/pjsor.v4i1.60.

[23] Sinha RR (2014) Estimation of ratio of two population means using auxiliary attributes in presence of non-response. International Journal on Recent Trends in Life Science and Mathematics, 1(2), 001007.

[24] Sinha RR \& Kumar V (2014) Improved classes of estimators for population mean using information on auxiliary character under double sampling the non-respondents. Nat Acad Sci Lett 37(1), 7179. http://dx.doi.org/10.1007/s40009-013-0198-z.

[25] Srivastava SK \& Jhajj HS (1983) a class of estimators of the population mean using multi-auxiliary information. Cal Stat Assoc Bull 32, 47-56.

[26] Srivastava S Rani, Srivastava SR \& Khare BB (1989) Chain ratiotype estimators for ratio of two population means using auxiliary characters. Commun Stat Theory Math 18 (10), 3917-3926. http://dx.doi.org/10.1080/03610928908830131. 